\title{
Los mapas mentales en el proceso de enseñanza y aprendizaje
}

\section{The mental maps in the teaching and learning process}

\author{
Eder Arturo Aco Corrales ${ }^{1,}$ \\ 1 Departamento Académico de Humanidades y Educación, Universidad Andina del Cusco, Cusco, Perú. \\ ”eaco@uandina.edu.pe
}

\begin{abstract}
Resumen
El objetivo del trabajo de la investigación fue describir la importancia de los mapas mentales en el proceso de enseñanza-aprendizaje; el trabajo corresponde a un nivel descriptivo, de diseño no experimental y enfoque cuantitativo. Para la recolección de datos se trabajó con 65 estudiantes del primer ciclo de la Facultad de Ciencias y Humanidades de la Universidad Andina del Cusco (entre 16, 17 y 18 años de edad de ambos sexos); se seleccionó esta población porque estos estudiantes desarrollan la asignatura de Técnicas de estudio y de investigación y en los contenidos se considera la elaboración de organizadores de la información (mapas mentales). Los resultados obtenidos mostraron que la elaboración de los mapas mentales son una técnica eficiente de organización para los estudiantes, lo que permite un adecuado manejo de la información y asimilación de conocimientos, que es muy alta, en comparación con otras técnicas o estrategias. Su uso en el contexto de enseñanza (docentes) es recomendado al momento de presentar información o exponer temas y también tiene un alto impacto en el proceso de aprendizaje (estudiantes), porque desarrolla el pensamiento creativo.
\end{abstract}

Palabras clave: Mapas mentales, enseñanza, aprendizaje, creatividad, información, organización.

\begin{abstract}
The present work developed aims to describe the importance of the use of mind maps in the teaching-learning process, the work corresponds to a descriptive level, non-experimental design and quantitative approach. To collect data, 65 students from the first cycle of the Faculty of Sciences and Humanities of the Andean University of Cusco were selected, this population was selected because these students develop the subject of study and research techniques and the contents of the subject is the development of visual organizers (mind maps). The results obtained show that the elaboration of mental maps is an efficient technique of organization for students, allows an adequate management of information and the assimilation of knowledge is very high compared to other techniques or strategies. Its use in the context of teaching (teachers) is recommended when presenting information or exposing topics and also has a high impact on the learning process (students), because it develops creative thinking.
\end{abstract}

Keywords: Mind maps, teaching, learning, creativity, information, organization.

Citar como: Corrales, EAA. (2019). Los mapas mentales en el proceso de enseñanza y aprendizaje, Rev Yachay, 8(1) ,559-565.

Recibido: 27-07-2019; Aceptado 21-09-2019; Publicado: 12-12-2019

\section{Introducción}

Muchas son las técnicas utilizadas para poder sistematizar la información, pero no todas se usan, sino que dependen de lo que se quiere lograr o de lo que se pretende estudiar. Es así que, de todas esas técnicas, una de las que tiene un alto impacto en el proceso de enseñanza - aprendizaje es el mapa mental.

El mapa mental es una técnica utilizada en el ámbito personal, familiar, educacional, empresarial, incluso es una técnica para tomar notas, realizar resúmenes, como herramienta nemotécnica o como medio para explicar ideas complejas.

No muchos estudiantes y docentes hacen uso de los mapas mentales, prefieren utilizar el subrayado, los resúmenes tipo ensayo y otros, que no tienen el mismo impacto. Se sabe que muchas de las técnicas mencionadas han marcado la etapa estudiantil en el caso de los docentes, dándonos grandes resultados; pero hoy en día se deben dar cambios significativos y dar paso a los organizadores visuales que, según estudios desarrollados en el campo de la psicología, producen mejores procesos cognitivos.

El objetivo fundamental del trabajo de investigación es describir la importancia de los mapas mentales en el proceso de enseñanza-aprendizaje. Los docentes deben hacer uso de diferentes estrategias para poder alcanzar los logros o resultados de aprendizaje planificados; una de esas tantas estrategias es la utilización de organizadores visuales, en los cuales se presenta el contenido de una manera diferente, rescatando aspectos importantes de un concepto o materia dentro de un esquema usando etiquetas. Pero dentro de todo 
ese grupo de organizadores existe uno que ha generado un alto impacto en el proceso de aprendizaje de los estudiantes como son los mapas mentales.

Las primeras muestras de lo que son los mapas mentales se dio aproximadamente en el siglo III D. C., cuando el pensador Porfirio de Tiro elaboró gráficamente conceptos que habían sido descritos por Aristóteles. Ya en el siglo XX aproximadamente, la teoría de los mapas de ideas se proyectó y se empezó a elaborar, utilizar y enseñar en las escuelas y no solo eso, sino que se incorporó también al mundo empresarial. A Tony Buzan, famoso psicólogo británico, se le considera como el promotor de los mapas en los negocios con la intensión de mejorar el pensamiento creativo, todo esto lo realizó en el siglo pasado.

De ese tiempo a la actualidad los mapas mentales han alcanzado un alto protagonismo en el desarrollo del proceso de enseñanzaaprendizaje, pero muy pocos docentes hacen uso de los mismos o fomentan en los estudiantes la elaboración de los mapas mentales. Estos son considerados por muchos estudiosos del campo de la Psicología como un método de análisis que permite organizar los pensamientos y hacer uso al máximo de las capacidades mentales (Buzan, 2004).

A diferencia de los otros organizadores visuales, los mapas mentales tienen una estructura más atractiva compuesta por ramificaciones que irradian de una imagen, palabra o símbolo central, hace uso de combinación de colores, en las ramificaciones también se puede utilizar palabras, símbolos y dibujos que se enlazan según un conjunto de reglas básicas, sencillas y amigables (Buzan, 2004).

Los mapas mentales permiten transformar una larga y aburrida lista de información o contenidos en diagramas atractivos y brillantes, permiten recordar con facilidad, sintetizan la información, son altamente organizados y están en sintonía con los procesos naturales del cerebro.

La utilidad que ofrecen los mapas mentales en el campo de la educación es más importante aún, porque su elaboración permite activar los procesos mentales y potencia el cerebro; los mapas mentales fomentan la creatividad, la retención y el aprendizaje en general. Son tan efectivos que se dice que los estudiantes que los usan son, en promedio, $75 \%$ más efectivo que lo normal (Chauvin, 2019).

La forma en que se presentan los mapas mentales, con símbolos, imágenes, palabras y combinaciones de colores, hacen de este organizador muy atractivo para los estudiantes, que hoy en día prefieren trabajar con esquemas gráficos que con textos cargados de información y difíciles de entender.

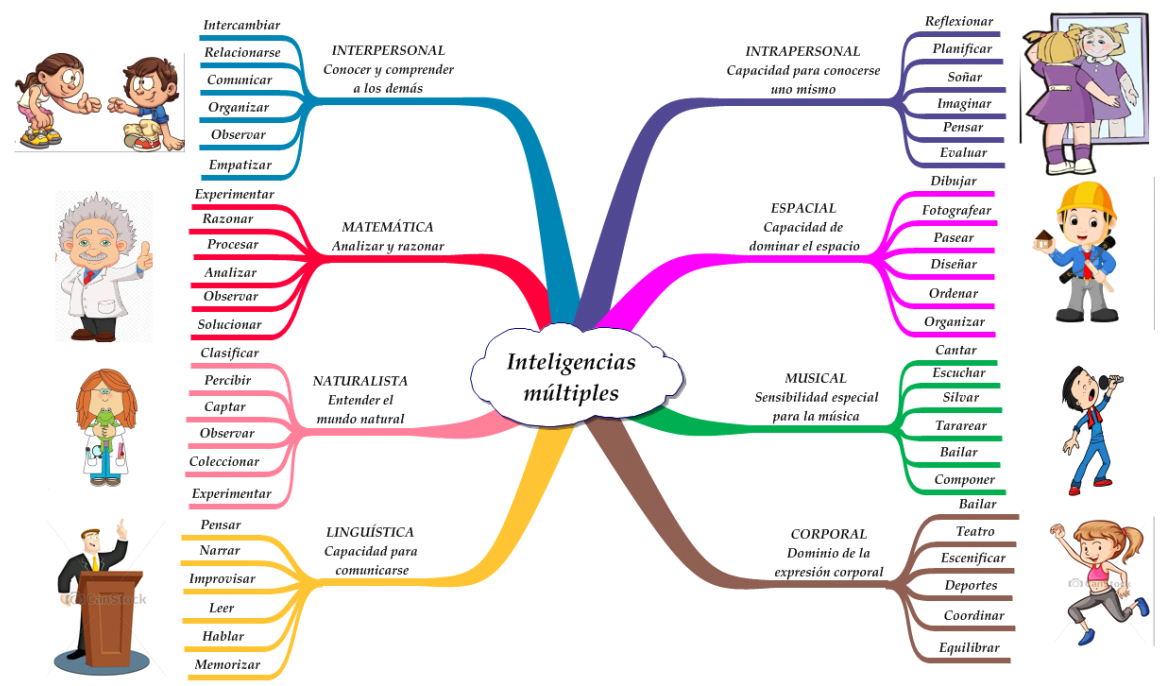

Figura 7: Mapa mental desarrollado por el autor para explicar la teoría de las inteligencias múltiples, desarrollado con el programa Inspiration.

Los mapas mentales no solo se vinculan con asignaturas de naturaleza teórica, sino que su elaboración también se relaciona con las asignaturas de matemáticas como lo demuestran los estudios realizados por Vega (2010), en su investigación "Influencia de los mapas mentales en el aprendizaje significativo de matemática en los alumnos del cuarto año de educación secundaria de la institución educativa 'Víctor Raúl Haya de la Torre' del Distrito Alto de la Alianza", donde demostró que el hacer uso de los mapas mentales ayuda en el 
aprendizaje de la asignatura de matemática. O el trabajo desarrollado por Rodas "Mapas mentales en el aprendizaje de las funciones trigonométricas", donde demostró que el uso de los mapas mentales influye en el aprendizaje de las funciones trigonométricas de los estudiantes.

Por lo que es necesario promover y fomentar la elaboración de los mapas mentales, los mismos que tienen un sello particular o propio de quien lo elabora, es una forma diferente y atractiva de tomar apuntes e incluso de presentar la información.

Desde el punto de vista de la evaluación, los mapas mentales son considerados como instrumentos de evaluación, porque su presentación nos brinda información valiosa sobre el desempeño de los estudiantes, ya que se puede evaluar: la técnica y presentación de la información, organización y asimilación de conocimientos; su proceso de evaluación como una actividad de aprendizaje se somete a la elaboración de una rúbrica, es sencillo de evaluar porque los mapas mentales se construyen en una sola página y es imposible que los estudiantes copien o presenten el mismo trabajo, debido a que cada mapa mental tiene un sello y característica personal.

\section{Materiales y método}

El presente estudio es de alcance descriptivo, de diseño no experimental y enfoque cuantitativo; la población estuvo conformada por estudiantes de la Facultad de Ciencias y Humanidades de la Universidad Andina del Cusco, el tipo de muestreo que se uso fue de tipo no probabilístico por criterio, se trabajó con 65 estudiantes. Para la recolección de datos se hizo uso de la técnica de la encuesta y se utilizó como instrumento un cuestionario conformado por 20 preguntas elaborado por Luis Víctor Ariste Cárdenas, trabajo desarrollado en la Universidad Nacional de Educación "Enrique Guzmán y Valle" en la ciudad de Lima (Ariste, 2013). Sobre el procesamiento de la información esta se realizó en el programa estadístico SPSS, se codificaron los datos y se extrajo la frecuencia para cada pregunta.

\section{Resultados}

Tabla 1:

Resumen de los resultados obtenidos al aplicar el cuestionario

\begin{tabular}{|c|c|c|c|c|c|c|c|c|c|c|c|}
\hline & \multirow{2}{*}{$\underset{\text { Ittems }}{N^{\circ}}$} & \multicolumn{2}{|c|}{$\begin{array}{l}\text { Totalmente } \\
\text { en } \\
\text { desacuerdo }\end{array}$} & \multicolumn{2}{|c|}{$\begin{array}{c}\text { Desacu } \\
\text { erdo }\end{array}$} & \multicolumn{2}{|c|}{ Indeciso } & \multicolumn{2}{|c|}{$\begin{array}{c}\text { De } \\
\text { acuerdo }\end{array}$} & \multicolumn{2}{|c|}{$\begin{array}{l}\text { Totalme } \\
\text { nte de } \\
\text { acuerdo }\end{array}$} \\
\hline & & $f$ & $\%$ & $f$ & $\%$ & $f$ & $\%$ & $f$ & $\%$ & $F$ & $\%$ \\
\hline 1 & $\begin{array}{l}\text { Aprendiste a usar } \\
\text { adecuadamente la técnica de } \\
\text { los mapas mentales. }\end{array}$ & 3 & 4.6 & 0 & 0 & 5 & 7.7 & 31 & 47.7 & 26 & 40 \\
\hline 2 & $\begin{array}{l}\text { La técnica del mapa mental te } \\
\text { ayuda a establecer la secuencia } \\
\text { lógica de un tema. }\end{array}$ & 2 & 3.1 & 0 & 0 & 4 & 6.2 & 36 & 55.4 & 23 & 35.4 \\
\hline 3 & $\begin{array}{l}\text { Expresas tu aprendizaje en } \\
\text { forma ordenada en un mapa } \\
\text { mental. }\end{array}$ & 2 & 3.1 & 0 & 0 & 12 & 18.5 & 36 & 55.4 & 15 & 23.1 \\
\hline 4 & $\begin{array}{l}\text { Los mapas mentales te ayudan } \\
\text { a registra sus saberes previos. }\end{array}$ & 2 & 3.1 & 0 & 0 & 4 & 6.2 & 50 & 76.9 & 9 & 13.8 \\
\hline 5 & $\begin{array}{l}\text { Al observar un mapa mental } \\
\text { puedes abstraer la ideal del } \\
\text { tema. }\end{array}$ & 1 & 1.5 & 0 & 0 & 7 & 10.8 & 38 & 58.5 & 19 & 29.2 \\
\hline 6 & $\begin{array}{l}\text { Utilizas organizadores del } \\
\text { conocimiento para elaborar un } \\
\text { mapa mental. }\end{array}$ & 1 & 1.5 & 0 & 0 & 13 & 20 & 41 & 63.1 & 10 & 15.4 \\
\hline 7 & $\begin{array}{l}\text { Te induce usar la creatividad } \\
\text { para elaborar un mapa mental. }\end{array}$ & 1 & 1.5 & 0 & 0 & 5 & 7.7 & 31 & 47.7 & 28 & 43.1 \\
\hline 8 & $\begin{array}{l}\text { Organizas tus ideas para } \\
\text { plasmarlo en un mapa mental. }\end{array}$ & 1 & 1.5 & 0 & 0 & 4 & 6.2 & 36 & 55.4 & 24 & 36.9 \\
\hline
\end{tabular}


9 Para elaborar un mapa mental debes asociar ideas de manera coherente.

10 Aplicas secuencialmente los niveles de comprensión cuando diseña un mapa mental.

11 Jerarquizas en forma adecuada los conceptos de un determinado tema.

12 Es posible que presentes un resumen de un contenido de un texto.

13 Cuándo te presentan un mapa mental puedes realizar una síntesis.

14 Manifiesta capacidad de análisis y síntesis en tus producciones.

15 Realizas análisis y síntesis de manera creativa cuando elaboras un mapa mental.

16 Un mapa mental te permite demostrar tus potencialidades y tu capacidad creativa.

17 Aporta conocimiento, habilidades y destrezas la realización de estructurar mapas mentales.

18 Estableces conceptos en base a tus experiencias intelectuales.

19 Comunicas asertiva y creativamente sus ideas a través de un mapa mental.

20 El manejo de la teoría es fundamental para le elaboración de un mapa mental.

Fuente: Aplicación del instrumento

Los resultados obtenidos muestran el alto impacto que tiene la elaboración de los mapas mentales en los procesos mentales, creatividad, manejo de la información, transmisión de ideas, jerarquización de conceptos, organización de información en los estudiantes.

La utilización de los mapas mentales generó una gran expectativa en los estudiantes, quienes, durante el semestre, fueron presentando mapas mentales mejor elaborados, más creativos y con las ideas principales y secundarias bien establecidas; la nueva tendencia de la educación nos empuja a poder desarrollar los procesos de sistematización de la información y los mapas mentales son una gran estrategia para alcanzar esa meta. 


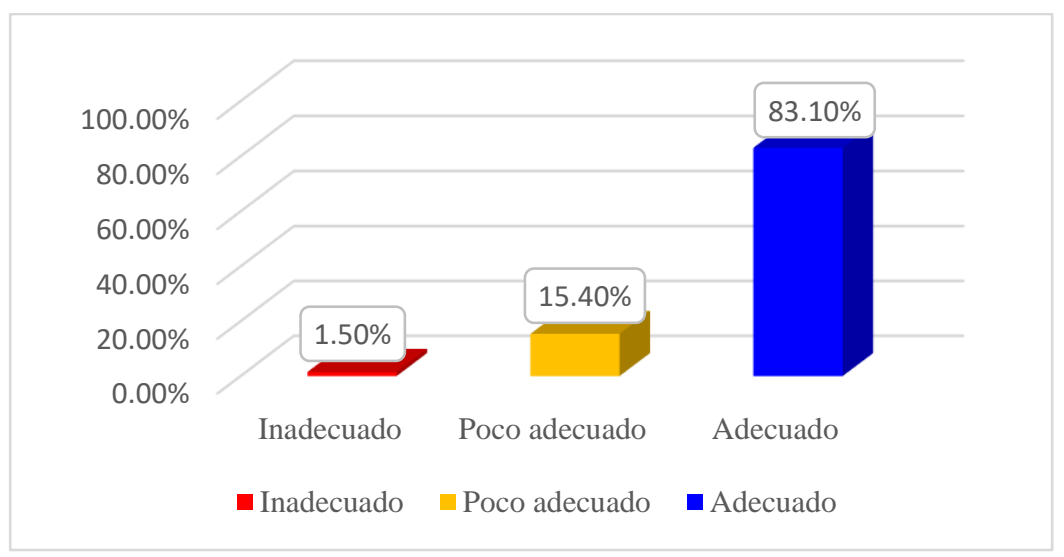

Figura 8: Resultados de la dimensión técnica de organización de la elaboración de los mapas mentales.

Según los resultados obtenidos el $83.10 \%$ de los estudiantes encuestados se muestra una adecuada técnica de organización de los mapas mentales; mientras que, el $15.4 \%$ muestra poco adecuada; y que sólo el 1.5\% muestra una adecuada técnica.

Los resultados muestran que para poder conseguir una adecuada organización de los temas desarrollados es imprescindible el uso de los mapas mentales, porque estos permitirán que los estudiantes manejen estructuras o esquemas que puedan aprender con mayor facilidad.

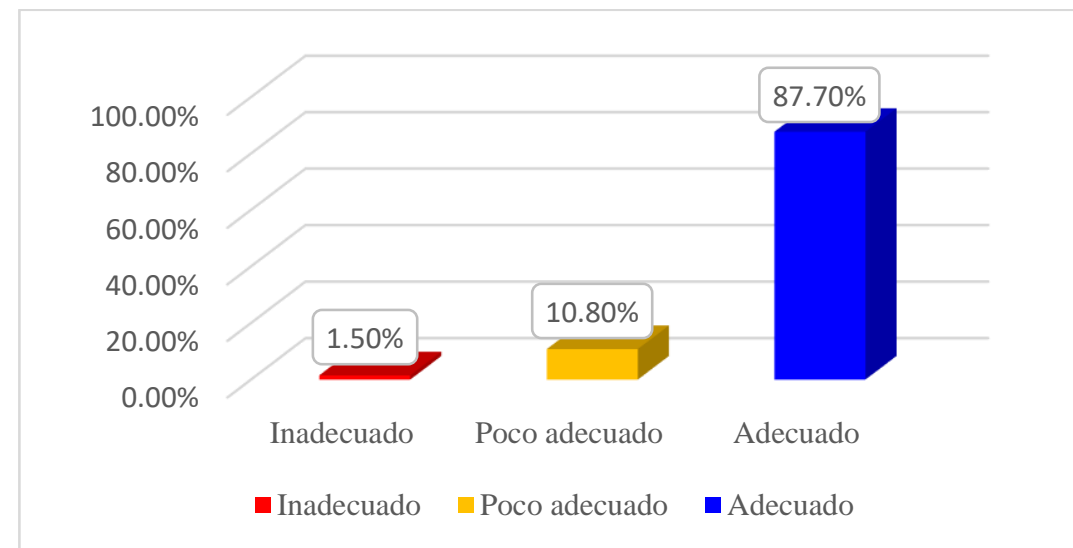

Figura 9: Resultados de la dimensión presentación de la información de la elaboración de los mapas mentales.

\section{Interpretación y análisis}

Según los resultados obtenidos, se puede observar que el $87.7 \%$ de los estudiantes encuestados muestran una adecuada presentación de la información al elaborar un mapa mental; mientras que, el $10.80 \%$ muestra poco adecuada y que, sólo el $1.5 \%$ de los estudiantes muestra inadecuada.

Los resultados indican que, al hacer uso de los mapas mentales, los estudiantes presentan adecuadamente la información, porque la esquematización y elaboración de los mapas mentales permiten la elección minuciosa de las palabras claves o ideas principales para su construcción. 


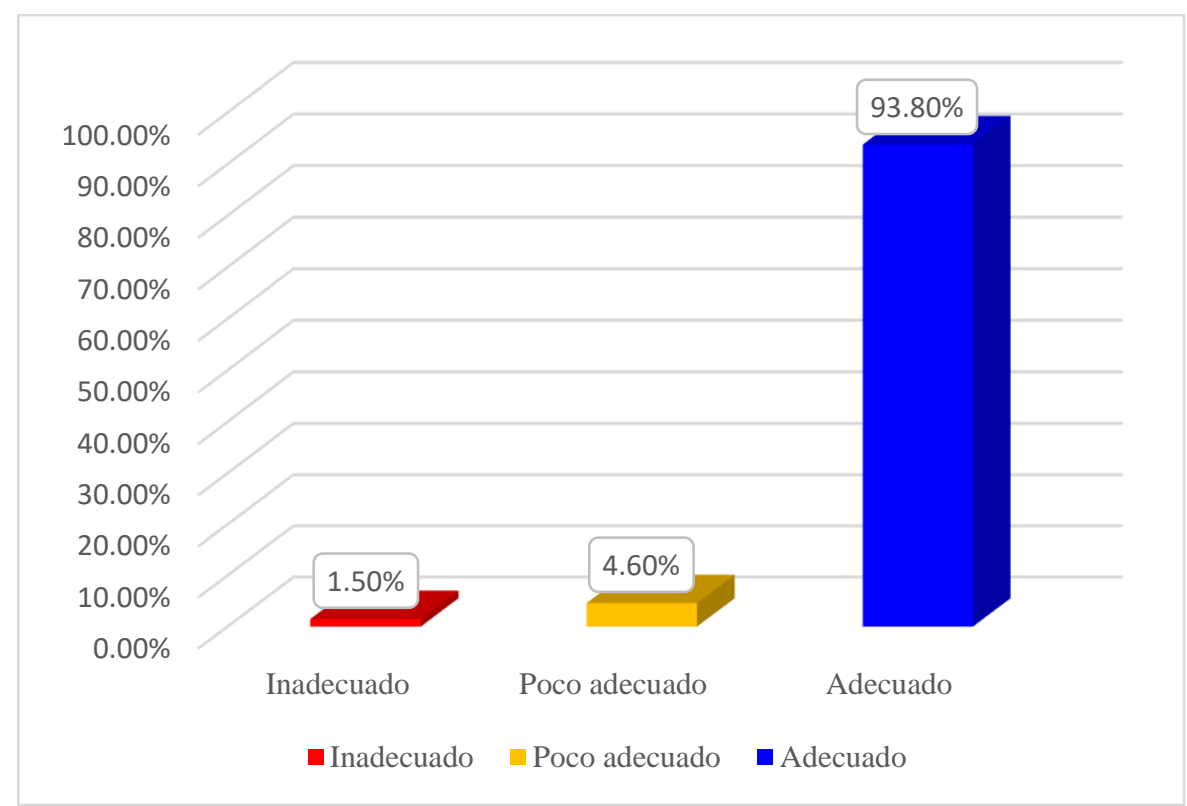

Figura 10. Resultados de la dimensión asimilación de los conocimientos de la elaboración de un mapa mental.

Los resultados obtenidos muestran que el $93.8 \%$ de los estudiantes encuestados muestran una adecuada asimilación de los conocimientos al elaborar un mapa mental; mientras que, el $4.6 \%$ de los estudiantes muestra poco adecuada; y que sólo el $1.5 \%$ de los estudiantes muestra inadecuada.

Los resultados obtenidos demuestran que el uso y elaboración de los mapas mentales desarrollan la capacidad de síntesis de contenidos textuales, también permite la asimilación de conocimientos. Estas estructuras visuales, permiten que los conceptos, definiciones, teorías y otros marcos teóricos sean asimilados por los estudiantes con mayor facilidad que con otras técnicas o métodos de estudio.

\section{Discusión}

Los resultados obtenidos en la elaboración y utilización de los mapas mentales es muy alta, estos resultados se asemejan a los obtenidos por Luis Víctor Ariste Cárdenas, trabajo desarrollado en la Universidad Nacional de Educación "Enrique Guzmán y Valle" en la ciudad de Lima, cuyo tema fue: "Aplicación de los mapas mentales y su influencia en el pensamiento creativo de los estudiantes del segundo grado de educación secundaria de la Institución Educativa pública "Andrés Bello", Pilcomayo-Huancayo", donde demostró que la elaboración de los mapas mentales, influyen en el pensamiento creativo de los estudiantes.

\section{Conclusiones}

Primera. El impacto de los mapas mentales en el proceso de enseñanza - aprendizaje es alto; muchas de la respuesta emitidas por los estudiantes muestran que, con la elaboración o construcción de los mapas mentales, organizan mejor los temas, presentan de una manera más atractiva los contenidos y su proceso de asimilación de conocimientos es mayor comparado con otras técnicas.

Segunda. La mayoría de los docentes presenta la información que no genera un alto impacto, pero esquematizando la información o estructurándola en algún tipo de organizador visual se obtendrán mejores resultados.

Tercera. La elaboración de los mapas mentales despierta el interés de los estudiantes al hacer uso de las palabras, símbolos, colores, gráficos, y dibujos. 
Cuarta. No solo es exigir a los estudiantes desarrollar un resumen, escribir un ensayo, elaborar una monografía; sino que el docente también debe cumplir las mismas tareas que exige a sus estudiantes, si se exige que se hagan mapas mentales para sistematizar la información y el docente no hace uso de los mismos, entonces qué tipo de enseñanza ofrecemos sino predicamos con el ejemplo.

\section{Referencias bibliográficas}

Ariste, L. (2013). Aplicación de los mapas mentales y su influencia en el pensamiento creativo de los estudiantes del segundo grado de educación secundaria de la Institución Educativa Pública "Andrés Bello", PilcomayoHuancayo. Lima: Universidad Enrique Guzman o Valle.

Ariza, Storino y Varela. (2017). El uso de los mapas mentales para el desarrollo del pensamiento crítico y reflexivo de estudiantes de $5^{\circ}, 8^{\circ}$ y $9^{\circ}$ en el reconocimiento de los cambios sociales de la historia. Barranquilla: Universidad del Norte.

Buzan, T. (2004). Cómo crear mapas mentales. Barcelona: Urano, S.A.

Caffaro, A. (2017). Mapas mentales y su incidencia en la comprensión lectora.
Quetzaltenango: Universidad Rafael Rodas, L. (2014). Mapas mentales en el Landívar.

Chauvin, S. (3 de Julio de 2019). Mujeres de empresa. Obtenido de Mapas mentales: http://www.mujeresdeempresa.com/map as-mentales-como-potenciar-nuestrocerebro-y-estimular-nuestra-creatividad/ López, C. (2015). Mapas mentales y su incidencia en el aprendizaje lógico. Quetzaltenango: Universidad Rafael Vega, Landívar.

López, R. (2014). El uso de los mapas mentales y su influencia en el aprendizaje de la filosofía en los estudiantes de grados décimo y undécimo de la Institución educativa Luis María Jiménez AguazulColombia, año 2014. Lima: Universidad Privada Norbert Wiener. aprendizaje de las funciones trigonométricas. Quetzaltenango: Universidad Rafael Landívar.

Rodríguez, E. M. (2008). Los mapas mentales como estrategia para favorecer la comprensión lectora en niños con TDAH Ajusco: Universidad pedagógica Nacional Unidad Ajusco.

, R. (2010). Influencia de los mapas mentales en el aprendizaje significativo de matemática en los alumnos del cuarto año de educación secundaria de la Institución Educativa "Víctor Raúla Haya de la Torre" del Distrito Alto de la Alianza en el año 2010. . Tacna: Universidad Nacional Jorge Basadre Grohmann-Tacna 